[white paper]

Diamond Open Access

\title{
Um Conjunto não Mensurável
}

\author{
Colaboração Matemática Aberta ${ }^{1}$ \\ 20 de Fevereiro de 2021
}

\begin{abstract}
Resumo
Uma das criações mais importantes da Análise do século XX foi a Integral de Lebesgue, que estendeu notavelmente a Integral de Riemann, resolveu em um período de poucos anos os problemas fundamentais da Teoria da Integração e deu um impulso relevante à Análise Funcional, à Teoria das Equações Diferenciais e Integrais e à Teoria da Probabilidade. 0 ponto básico dessa nova teoria foi a introdução da noção de medida. Medida, a grosso modo, é uma função cujo domínio é um conjunto de subconjuntos de $\mathbb{R}$ e cujo contradomínio é o conjunto dos números reais não negativos (unido com o símbolo $+\infty$ ). 0 comprimento de um intervalo, por exemplo, é uma medida, digamos $L$, definida sobre todos os intervalos da reta real, tal que $L(I)=b-a$, onde $a$ e $b$ são os extremos do intervalo $L$ e $L(I)=+\infty$ se $L$ for não limitado. Ora, a medida $L$ está definida apenas para intervalos. Seria interessante estender esse conceito para outros subconjuntos da reta. Neste trabalho, será definida a medida exterior de um subconjunto de $\mathbb{R}$ e, com esta medida, será definida a noção de conjunto mensurável.
\end{abstract}

palavras-chave: Lebesgue, conjunto mensurável, medida A versão mais atualizada deste artigo está disponivel em https://osf.io/935q8/download

${ }^{1}$ Todos os autores com suas afiliações aparecem no final deste artigo. 


\section{Introdução}

1. Iremos definir a medida exterior de um conjunto e alguns resultados importantes dessa definição.

2. Apresentaremos, também, o conceito de medida.

3. O leitor não especialista nos conceitos de medida e integração pode consultar [1-3].

\section{A Medida}

4. A reta real estendida é o conjunto $\mathbb{R}^{ \pm \infty}=\mathbb{R} \cup\{ \pm \infty\}$.

5. Seja I qualquer intervalo de números reais estendidos.

6. O comprimento $L(I)$ de $I$ é definido como sendo o módulo da diferença entre seus pontos extremos.

7. A função comprimento é um exemplo de uma função de conjuntos, cujo domínio é o conjunto de todos os intervalos de uma reta.

8. Gostaríamos, então, de estender (6) para comprimento de diferentes conjuntos de intervalos.

9. Vamos definir uma função de conjuntos $m$ tal que para todo conjunto $E$ de uma certa coleção $M$ de conjuntos de números reais corresponda a um número real estendido não negativo $m E$ chamado a medida de E.

10. Seria ideal que as propriedades (11)-(14) a seguir fossem satisfeitas pela função $m$.

11. $m E$ está definida para todo conjunto $E$ de números reais, isto é, o domínio de $m$ é $\wp(\mathbb{R})$, o conjunto das partes de $\mathbb{R}$.

12. Para qualquer intervalo $I, m I=L(I)$. 
13. Se $\left\langle E_{n}\right\rangle$ é uma sequência de conjuntos disjuntos, então

$$
m\left(\cup E_{n}\right)=\sum\left(m E_{n}\right),
$$

isto é, a medida da união é a soma das medidas.

14. $m$ é invariante por translação, isto é, se $E$ é um conjunto para o qual $m$ está definida e se $E+y=\{x+y ; x \in E\}$ é o conjunto obtido pela substituição do ponto $x$ em $E$ pelo ponto $x+y$ com $y \in \mathbb{R}^{ \pm \infty}$, então

$$
m(E+y)=m E .
$$

15. De fato, considere uma cobertura de $E$.

16. De (27),

$$
m^{*}(E)=\inf \sum L\left(I_{n}\right)
$$

17. Se $\left(I_{n}\right)$ cobre $E$, então $\left(I_{n}+y\right)$ cobre $E+y$.

18. E, se $\left(J_{n}\right)$ cobre $E+y$, então $\left(J_{n}-y\right)$ cobre $E$.

19. Além disso, sabemos que

$$
L\left(I_{n}\right)=L\left(I_{n}+y\right) \quad \text { e } \quad L\left(J_{n}\right)=L\left(J_{n}-y\right) .
$$

20. Segue que

$$
\inf _{e \subset I_{n}} \sum L\left(I_{n}\right)=\inf _{E+y \subset J_{n}} \sum L\left(J_{n}\right),
$$

isto é,

$$
m^{*}(E)=m^{*}(E+y), \quad \forall y \in \mathbb{R}^{ \pm \infty} .
$$

21. No entanto, é impossível construir $m$ que satisfaça todas as propriedades (11)-(14) (veja [4]). 


\section{Medida Exterior}

22. Seja $A$ um conjunto qualquer de números reais.

23. Defina $I_{n}$ para todo $n \in \mathbb{N}=\{1,2,3, \ldots\}$.

24. Considere a coleção de intervalos $I_{n}$ a qual cobre $A$, isto é, $A \subset \cup I_{n}$, com $n \in \mathbb{N}$.

25. Considere, também, a soma dos comprimentos de cada $I_{n}$.

26. Uma vez que o comprimento de um intervalo é um número positivo, esta soma está unicamente determinada não importando a ordem de seus termos.

27. Definimos, então, a medida exterior de $A$ como sendo

$$
m^{*} A=\inf _{A \subset \cup I_{n}} \sum L\left(I_{n}\right) .
$$

28. O lado direito de (27) é o ínfimo da soma dos comprimentos da coleção de intervalos $I_{n}$ a qual cobre o conjunto $A$.

29. Veja que $m^{*} \varnothing=0$ e se $A \subset B$, então $m^{*} A \leq m^{*} B$. [4]

30. Se $A$ contém um único ponto, então $A$ tem medida exterior zero. [4]

31. Se $I$ é um intervalo qualquer, então $m^{*} I=L(I)$. [4]

32. Seja $\left\{A_{n}\right\}$ uma coleção enumerável de subconjuntos de $\mathbb{R}$. Então

$$
m^{*}\left(\bigcup A_{n}\right) \leq \sum m^{*} A_{n} .
$$

\section{Conjuntos Mensuráveis}

33. A função $m^{*}$ está definida para qualquer conjunto da reta.

34. Nem sempre a propriedade (13) será satisfeita devido a (31) e (32). 
35. Um conjunto $E$ é mensurável se para cada conjunto $A$ tivermos

$$
m^{*} A=m^{*}(A \cap E)+m^{*}\left(A \cap E^{c}\right) .
$$

36. A Fig. 1 nos ajuda a compreender melhor (35).

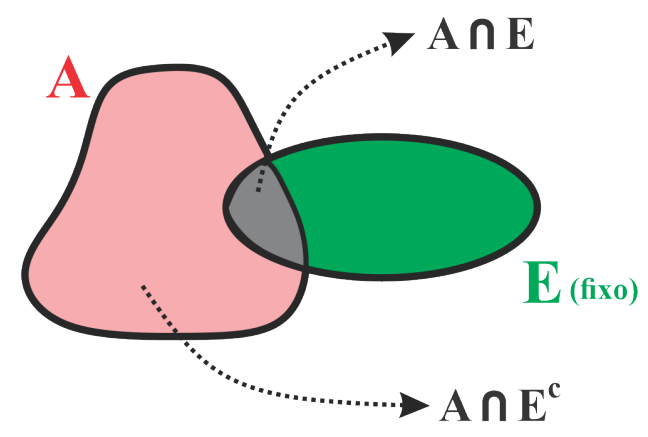

Figura 1: Conjunto Mensurável E.

\section{Teorema}

37. A coleção $M$ de conjuntos mensuráveis é uma $\sigma$-álgebra. [4]

\section{Proposição 1}

38. Seja $\left\langle E_{n}\right\rangle$ uma sequência de conjuntos mensuráveis. Então [4]

$$
m\left(\cup E_{n}\right) \leq \sum m E_{n} .
$$

39. Se os conjuntos $E_{n}$ são dois a dois disjuntos, então

$$
m\left(\cup E_{n}\right)=\sum m E_{n}
$$

\section{Lema 1}

40. Seja $E \subset[0,1]$ um conjunto mensurável. [4]

41. Então para cada $y \in[0,1]$, o conjunto $E+y$ é mensurável e

$$
m(E+y)=m E .
$$




\section{Um Conjunto não Mensurável}

42. Considere, no intervalo $[0,1] \subset \mathbb{R}$, a seguinte relação:

$$
x, y \in[0,1], x \sim y \Leftrightarrow x-y \text { é racional. }
$$

43. Note que $\sim$ define uma relação de equivalência no conjunto $[0,1]$.

44. Escolhemos um representante de cada classe de equivalência de $\sim$ por meio do Axioma da Escolha.

45. Chamemos de $V$ o conjunto formado pelos representantes de (44).

46. Considere $\left\{r_{j}\right\}_{j=1}^{\infty}$ uma enumeração do conjunto $[-1,1] \cap \mathbb{Q}$.

47. Defina

$$
S=\bigcup_{j=1}^{\infty}\left(V+r_{j}\right), \quad \text { onde } V+r_{j}=x+r_{j}: x \in V .
$$

48. Veja que as seguintes inclusões são verdadeiras:

$$
[0,1] \subseteq S \subseteq[-1,2]
$$

49. Pela construção do conjunto $V$, existe $y \in V$ tal que $r=x-y \in \mathbb{Q}$.

50. Como $x, y \in[0,1]$, então $x-y=r \in[-1,1]$.

51. Logo, existe $j \in \mathbb{N}$ tal que $r=r_{j}$.

52. Isso mostra que $x=y+r_{j} \in\left(V+r_{j}\right) \subset S$. Daí $[0,1] \subset S$.

53. Agora, seja $x \in S$, isto é, $x=y+r_{j}$ para algum $j \in \mathbb{N}$ e $y \in V$.

54. Como $r_{j} \in[-1,1]$ e $y \in V \subset[0,1]$, a soma $y+r_{j} \in[-1,2]$.

55. Portanto, $x \in[-1,2]$ e as inclusões (48) estão provadas.

56. Os conjuntos $\left\{V+r_{j}: j \in \mathbb{N}\right\}$ são dois a dois disjuntos. 
57. Facilmente prova-se (56).

58. Suponha agora, por absurdo, que $V$ seja mensurável.

59. Decorre da Proposição 1 (38) e do Lema 1 (40) que

$$
\begin{aligned}
{[0,1] \subseteq S \subseteq[-1,2] } & \Rightarrow m([0,1]) \leq m(S) \leq m([-1,2]) \\
& \Rightarrow 1 \leq \sum_{j=1}^{\infty} m(V) \leq 3 .
\end{aligned}
$$

60. Note que o somatório de (59) pode assumir apenas dois valores, a saber, zero, quando $m(V)=0$, ou infinito, quando $m(V)>0$.

61. Mas isso é um absurdo, pois essa soma está entre 1 e 3.

62. A contradição se deve ao fato de admitir que $V$ é mensurável.

\section{Considerações Finais}

63. Assim, fica provado que $V$ é um conjunto não mensurável.

\section{Ciência Aberta}

O arquivo latex para este artigo, juntamente com outros arquivossuplementares, estão disponíveis em [5].

\section{Consentimento}

64. O autor concorda com [6]. 


\section{Referências}

[1] Bartle, R. G. Elements of integration. John Wiley and Sons. NewYork. 1966.

[2] Royden, M. Real Analysis. MacMillan, New York, 1963.

[3] Rudin, W. Real and Complex Analysis. Mc-Graw Hill, New York, 1966.

[4] Fernandez, P. J. Medida e Integração. Rio de Janeiro: IMPA, 1986.

[5] Lobo, Matheus P. "Open Journal of Mathematics and Physics (OJMP)." OSF, 21 Apr. 2020.

https://doi.org/10.17605/osf.io/6hzyp

https://osf .io/6hzyp/files

[6] Lobo, Matheus P. "Simple Guidelines for Authors: Open Journal of Mathematics and Physics." OSF Preprints, 15 Nov. 2019.

https://doi.org/10.31219/osf.io/fk836

\section{Colaboração Matemática Aberta}

\section{Eduardo Dias Lima ${ }^{1}$}

(autor principal, duardo.dias16@hotmail.com)

${ }^{1}$ Universidade Federal de Goiás (Brasil) 